\title{
Reduced functional connectivity between bilateral precuneus and contralateral parahippocampus in schizotypal personality disorder
}

Yikang Zhu ${ }^{1,2 \dagger}$, Yunxiang Tang ${ }^{3 \dagger}$, Tianhong Zhang ${ }^{1}$, Hui Li ${ }^{1}$, Yingying Tang ${ }^{1}$, Chunbo Li ${ }^{1,6}$, Xingguang Luo ${ }^{4}$, Yongguang $\mathrm{He}^{1^{*}}$, Zheng $\mathrm{Lu}^{5^{*}}$ and Jijun Wang ${ }^{1,6}$

\begin{abstract}
Background: Schizotypal personality disorder (SPD) is linked to schizophrenia in terms of shared genetics, biological markers and phenomenological characteristics. In the current study, we aimed to determine whether the previously reported altered functional connectivity (FC) with precuneus in patients with schizophrenia could be extended to individuals with SPD.

Methods: Twenty subjects with SPD and 19 healthy controls were recruited from 4461 freshmen at a university in Shanghai and received a resting-state scan of MRI. All participants were evaluated by the Chinese version of Schizotypal Personality Questionnaire (SPQ) and the Chinese version of Symptom Checklist (SCL-90). The imaging data were analysed using the seed-based functional connectivity method.

Results: Compared with the controls, SPD subjects exhibited reduced FC between bilateral precuneus and contralateral parahippocampus. In SPD group, SPQ total score was negatively correlated with FC between right precuneus and left parahippocampus $(r=-0.603, p=0.006)$; there was a negative trend between SPQ subscale score of suspiciousness and FC between left precuneus and right parahippocampus ( $r=-0.553, p=0.014)$; and a positive trend was found between SPQ subscale score of odd or eccentric behaviour and FC between left precuneus and right superior temporal gyrus $(r=0.543, p=0.016)$. As for the SCL-90 score, a similar negative trend was found between SCL-90 subscale score of suspiciousness and FC between right precuneus and left parahippocampus $(r=-0.535, p=0.018)$ in SPD group.

Conclusions: Our findings suggest that the decreased functional connectivity between precuneus and contralateral parahippocampus might play a key role in the pathophysiology of schizophrenia spectrum disorder.
\end{abstract}

Keywords: Schizotypal personality disorder, Functional connectivity, Resting state, Precuneus, Parahippocampus

\footnotetext{
*Correspondence: hyg_512@126.com; luzheng@tongji.edu.cn

${ }^{\dagger}$ Equal contributors

${ }^{1}$ Shanghai Key Laboratory of Psychotic Disorders, Shanghai Mental Health

Center, Shanghai Jiao Tong University School of Medicine, South Wan Ping

Road 600, Shanghai 200030, People's Republic of China

${ }^{5}$ Department of Psychiatry, Shanghai Tongji Hospital, Tongji University School

of Medicine, 389 Xin Cun Road, Shanghai 200065, People's Republic of China

Full list of author information is available at the end of the article
} 


\section{Background}

Schizotypal personality disorder (SPD) is categorized as a schizophrenia spectrum disorder in the DSM-5 [1]. It is linked to schizophrenia in terms of shared genetics, biological markers and phenomenological characteristics [2]. Although individuals with SPD experience less severe psychotic-like symptoms, they exhibit an array of cognitive dysfunctions that are qualitatively similar to those observed in schizophrenia, such as attention and information processing, working memory and executive functioning [3-7]. Thus studying individuals with SPD can be a powerful strategy for advancing knowledge regarding the pathophysiology of schizophrenia. Moreover, SPD individuals are often unmedicated and are rarely in acute episode, which offers an apparent advantage in avoiding such confounding factors.

Precuneus is increasingly obtaining attention from researchers in the neuroimaging studies of psychiatric disorders [8-14]. It is located in the medial area of the superior parietal cortex and is involved in episodic memory, visuospatial processing, reflections upon self and aspects of consciousness [15-17]. Converging evidence suggests that the precuneus is a functional core of the default network $[18,19]$. The latest view is that there is a dynamic model of cross lifespan functional segregation and integration between precuneus-dorsal posterior cingulate cortex network and default network [20], which highlights the importance of this region in the neurodevelopment. Structural and functional abnormalities of the precuneus were frequently reported in many neurological and psychiatric conditions including Alzheimer's disease and mild cognitive impairment [21-30], and depression [31-36]. In schizophrenia, several studies reported significant abnormalities in functional connectivity (FC) of precuneus. The study by Kraguljac et al. [37] found deficits in resting state functional connectivity between precuneus and hippocampus, and glutamate abnormalities in the hippocampus in unmedicated patients with schizophrenia. Although the authors did not observe a direct relationship between these abnormalities, their findings partly elucidated the brain structures of the functional connectivity alterations in schizophrenia. The study by Gong et al. [10] provided genetic evidence of functional connectivity of precuneus. They found that both precuneus and its functional connectivity could be linked with DISC1 polymorphisms, which plays a role in both neural signalling and neurodevelopment and have been associated with schizophrenia. In first episode schizophrenia, another study by Guo et al. [11] found that FC between precuneus and bilateral Heschl's gyri was not only abnormal, but also its correlation with a cognitive component of event-related potentials (ERPs), P300 amplitude, was absent. Although no study reported FC of precuneus in SPD, Nenadic et al. [38] did found the positive correlation of right precuneus with negative schizotypy scores among 59 healthy non-clinical volunteers using voxel-based morphometry (VBM). Therefore, precuneus is one of key areas for us to understanding schizophrenia spectrum disorders.

In the present study, we explored functional connectivity across whole brain with precuneus as the seed region in undergraduate students with schizotypal personality traits. We hypothesized that precuneus FC would be altered in these SPD subjects and could be associated with their schizotypal traits or subclinical symptoms.

\section{Methods \\ Participants}

Twenty subjects with SPD and 19 HCs were recruited from 4461 freshmen at a university in Shanghai. All participants were evaluated by a 74-item scale of the Chinese version of Schizotypal Personality Questionnaire (SPQ) and a 90 -item scale of the Chinese version of Symptom Checklist (SCL-90). The Schizotypal Personality Questionnaire (SPQ) is a popular method of measuring both normal variability and abnormal degrees of schizotypy [39]. It has 9 subscales, including ideas of reference, social anxiety, odd beliefs/magical thinking, unusual perceptual experiences, eccentric/odd behavior and appearance, no close friends, odd speech, constricted affect, suspiciousness/ paranoid ideation. For schizotypy and SPQ, the three higher-order factors proposed are Cognitive Perceptual (Ideas of Reference/Suspiciousness, Magical Thinking, and Unusual Perceptions), Interpersonal (No Close Friends/ Constricted Affect and Social Anxiety), and Disorganized (Eccentric Behavior and Odd Speech) [40]. The Symptom Checklist-90 (SCL-90) is a questionnaire that is widely applied self-assessment tool for individuals with a broad range of mental disorders and symptom intensity [41]. It contains 90 items, yielding nine scores for primary symptom dimensions and three for global distress. The symptom dimensions comprise somatization, obsessive-compulsive behavior, interpersonal sensitivity, depression, anxiety, hostility, phobic anxiety, paranoid ideation (Suspiciousness), and psychoticism. The main global index of distress is the global severity index (GSI), which is the average of all responses. The test-retest reliability of the Chinese version SPQ after 4 weeks was 0.84 [42]. We defined top $10 \%$ of SPQ total scores in our screened sample as high SPQ scores. These eligible subjects were further interviewed by senior psychiatrists with Structured Clinical Interview for DSM-IV Axis II (SCID-II), and all the subjects in the SPD group in the present study met DSM-IV diagnostic criteria for SPD. The control subjects of SPD were randomly selected from those participants with low 10\% of SPQ total scores. Both SPD group and control group underwent brain magnetic resonance imaging (MRI), and their resting-state functional MRI (rs-fMRI) data was collected. Three of them 
were excluded because of the poor quality of MRI scanning, and whose background data were not include in this report. The study was approved by the local institutional ethics board (Institutional Review Board of Shanghai Mental Health Center, Shanghai Jiao Tong University School of Medicine) and carried out in accordance with the Declaration of Helsinki. Written informed consent was obtained from each participant prior to inclusion.

\section{MRI acquisition}

All images were acquired on a 3.0-T MR scanner (Trio, Siemens, Erlangen, Germany). A high-resolution T1weighted sequence with the following parameters was used: repetition time $(\mathrm{TR})=1900 \mathrm{~ms}$, echo time $(\mathrm{TE})=2.46 \mathrm{~ms}$, flip angle $=9^{\circ}$, field of view $(\mathrm{FOV})=256 \times 256 \mathrm{~mm} 2$, slice thickness $=1 \mathrm{~mm}$ and 192 slices. The rs-fMRI data were acquired using a gradient echo-planar imaging pulse sequence with the following parameters: $\mathrm{TR}=2000 \mathrm{~ms}$, $\mathrm{TE}=25 \mathrm{~ms}$, flip angle $=90^{\circ}, \mathrm{FOV}=240 \times 240 \mathrm{~mm} 2$, voxel size $=3.446 \times 3.446 \times 5 \mathrm{~mm} 3$, slice gap $=0$. Each brain volume comprised 32 axial slices. Participants were asked to lie down and remain motionless, keep eyes open and fixation on a cross on the screen during the scan.

\section{Image processing}

The rs-fMRI preprocessing was carried out using Data Processing Assistant for Resting-State fMRI (DPARSF version 2.0, http://www.restfmri.net). After the first 10 volumes were discarded, the images were corrected for slice timing and head motion. The data with over $3^{\circ}$ or $3 \mathrm{~mm}$ movement were excluded from further analysis. The functional images were normalized to standard Montreal Neurological Institute (MNI) space and resampled to $3 \times 3 \times 3 \mathrm{~mm} 3$. Following the spatial smooth with a 4-mm full width at half-maximum (FWHM) Gaussian kernel, data were temporally band-pass filtered $(0.01-0.08 \mathrm{~Hz})$ and linearly detrended. The final step of preprocessing was linear regression to remove several sources of spurious covariates, including the effect of the average signals from white matter region, cerebral spinal fluid (CSF) region and whole brain signals, and the six head-motion parameters. We used the Resting-state fMRI Data Analyze Toolkit (Rest version 1.6, http://www.restfmri.net) to calculate the functional connectivity (FC) with bilateral precuneus as region-ofinterest (ROIs). The two ROIs of bilateral precuneus were identified by an AAL template. Through computing the Pearson correlation coefficients between the time course of ROI voxel and that of all other brain voxels, we obtained the results of cross-correlation FC analysis. This was followed by normalization to Z-scores from correlation coefficients for each voxel with Fisher's transformation. Finally, we extracted the individual Z-score maps for the preparation of group analysis.

\section{Statistical analysis}

We also used the Resting-state fMRI Data Analyze Toolkit (Rest version 1.6, http://www.restfmri.net) to perform the group analysis. An independent $t$-test analysis was conducted with a significant threshold of $p<0.001$ at the voxel level and $p<0.05$ at the cluster scale (corrected for multiple comparisons using Monte Carlo simulation with the program Alphasim in AFNI). Then, we extracted the average Z-scores from each significantly different FC cluster with bilateral precuneus between two groups to perform two-tailed Pearson correlation analysis with SPQ or SCL-90 scores. The level of significance was set to $p<0.01$.

\section{Results \\ Demographic and clinical assessment}

The demographic and clinical assessment of control and SPD groups were shown in Table 1. No significant difference was found between two groups in age and gender. All participants were undergraduate students. The SPQ scores and SCL-90 score in SPD group were significantly higher than those in the control group $(p<0.001)$.

\section{Group differences in FC of bilateral precuneus}

Compared with control group, SPD group showed decreased FC between right precuneus and bilateral parahippocampus, decreased FC between right precuneus and right middle temporal gyrus, but increased FC between right precuneus and right middle frontal gyrus. As for left precuneus, SPD group only showed reduced FC with right parahippocampus and right superior temporal gyrus (see Table 2 and Fig. 1).

\section{Correlations of FC of precuneus with SPQ score and SCL-90 score}

In SPD group, SPQ total score was found to be negatively correlated with FC between right precuneus and left parahippocampus $(r=-0.603, p=0.006)$; there was a negative trend between SPQ subscale score of suspiciousness and FC between left precuneus and right parahippocampus $(r=-0.553, p=0.014)$; and a positive trend was found between SPQ subscale score of odd or eccentric behaviour and FC between left precuneus and right superior temporal gyrus $(r=0.543, p=0.016)$. As for the SCL-90 score, a similar negative trend was found between SCL-90 subscale score of paranoid ideation and FC between right precuneus and left parahippocampus $(r=-0.535, p=0.018)$ in SPD group (see Fig. 2). In control group, a positive correlation was found between SPQ subscale score of constricted affect and FC between right precuneus and right middle temporal gyrus $(r=0.670, p=0.003)$. 
Table 1 Demographic and clinical assessment

\begin{tabular}{|c|c|c|c|}
\hline Variable (mean \pm S.D.) & $\begin{array}{l}\text { Control group } \\
(n=17)\end{array}$ & $\begin{array}{l}\text { SPD group } \\
(n=19)\end{array}$ & $P$ \\
\hline Age (years) & $19.71 \pm 0.71$ & $19.98 \pm 0.82$ & 0.305 \\
\hline Gender (M/F), n & $16 / 1$ & $17 / 2$ & - \\
\hline SCL-90 total score & $24.82 \pm 22.18$ & $114.42 \pm 65.83$ & $<0.001^{*}$ \\
\hline SPD total score & $11.71 \pm 4.09$ & $47.42 \pm 9.37$ & $<0.001^{*}$ \\
\hline \multicolumn{4}{|l|}{ SPD subscale: } \\
\hline 1. Ideas of Reference & $2.76 \pm 1.60$ & $7.11 \pm 1.94$ & $<0.001^{*}$ \\
\hline 2. Social Anxiety & $0.65 \pm 0.93$ & $5.58 \pm 1.84$ & $<0.001^{*}$ \\
\hline $\begin{array}{l}\text { 3. Odd Beliefs/Magical } \\
\text { Thinking }\end{array}$ & $1.29 \pm 1.21$ & $3.89 \pm 1.76$ & $<0.001^{*}$ \\
\hline $\begin{array}{l}\text { 4. Unusual Perceptual } \\
\text { Experiences }\end{array}$ & $1.12 \pm 1.65$ & $5.84 \pm 2.63$ & $<0.001^{*}$ \\
\hline $\begin{array}{l}\text { 5. Eccentric/Odd Behavior } \\
\text { and Appearance }\end{array}$ & $0.59 \pm 0.71$ & $4.26 \pm 1.88$ & $<0.001^{*}$ \\
\hline 6. No Close Friends & $0.94 \pm 1.09$ & $4.32 \pm 2.21$ & $<0.001^{*}$ \\
\hline 7. Odd Speech & $2.00 \pm 1.00$ & $6.95 \pm 1.55$ & $<0.001^{*}$ \\
\hline 8. Constricted Affect & $1.59 \pm 2.00$ & $4.42 \pm 2.04$ & $<0.001^{*}$ \\
\hline $\begin{array}{l}\text { 9. Suspiciousness/Paranoid } \\
\text { Ideation }\end{array}$ & $0.76 \pm 0.83$ & $5.05 \pm 2.48$ & $<0.001^{*}$ \\
\hline
\end{tabular}

S.D. standard deviation, $M$ male, $F$ female, $S P Q$ schizotypal personality questionnaire, SPD schizotypal personality disorder

${ }^{*} p<0.05$ significant two-sided testing

\section{Discussion}

This is the first study of FC of precuneus in SPD. In the present study, 20 SCID-II diagnosed SPD subjects from a pool of 4461 first year college students were compared with 19 subjects of low SPQ score and bilateral precuneus were selected as ROIs in functional connectivity analysis of resting state MRI scan. There were two main findings. First, FC of right precuneus with bilateral parahippocampus and right middle temporal gyrus, FC of left precuneus with right parahippocampus and right superior temporal gyrus, was reduced in SPD group as compared to the control group. There is an increased FC of right precuneus with right middle frontal gyrus in SPD subjects. Second, in the SPD group, individuals with higher SPQ total scores and SCL-90 subscale score of paranoid ideation showed stronger reduction in FC between right precuneus and left parahippocampus. These results indicate the fronto-parietal and temporal-parietal connection alterations in the unmedicated individuals with SPD and associations of hippocampal-parietal FC reduction with schizotypal traits.

The precuneus plays a pivotal role in a wide spectrum of highly integrated tasks, including visuo-spatial imagery, episodic memory retrieval and self-processing operations [15]. Furthermore, the precuneus is regarded as a hub in the functional connectome, also as part of the default mode network $[18,19]$. As expected, we found that the altered FC of precuneus among the individuals with SPD. During the resting state, the control group showed reduced fronto-parietal and increased temporalparietal connections, relative to the SPD group. One possible interpretation is that activity in the fronto-parietal control network (FPC) decrease and activity in the default mode network (DMN) increase. It has been well documented that the FPC-DMN anti-correlation is affected in schizophrenia patients [43-46]. The present study found a reduced FC between right precuneus and right middle temporal gyrus, but an increased FC between right precuneus and right middle frontal gyrus, supporting that the FPC-DMN anti-correlation is also affected among SPD subjects. Recently, Xin et al. [47] found that individual differences in empathic ability were significantly correlated with the FPC-DMN anti-correlation.

We further found that a reduced FC between right precuneus and left parahippocampus was significantly correlated with SPQ total scores and SCL-90 subscale score of paranoid ideation among SPD patients. Kraguljac et al. [37] reported hippocampal-parietal dysconnectivity

Table 2 Group differences in functional connectivity of bilateral precuneus

\begin{tabular}{|c|c|c|c|c|c|c|c|}
\hline \multirow[t]{2}{*}{ Direction } & \multirow[t]{2}{*}{ Region } & \multirow{2}{*}{$\begin{array}{l}\text { Cluster } \\
\text { size }\end{array}$} & \multicolumn{3}{|c|}{ Peak coordinates } & \multirow{2}{*}{$\begin{array}{l}\mathrm{SPD}^{\mathrm{a}} \\
\text { mean }(\mathrm{sd})\end{array}$} & \multirow{2}{*}{$\begin{array}{l}\mathrm{HC}^{\mathrm{a}} \\
\text { mean }(\mathrm{sd})\end{array}$} \\
\hline & & & $x$ & y & z & & \\
\hline \multicolumn{8}{|l|}{ PCUN.R } \\
\hline \multirow[t]{3}{*}{ control > SPD } & PHG.L & 16 & -21 & -27 & -27 & $-0.065(0.092)$ & $0.151(0.101)$ \\
\hline & PHG.R & 9 & 39 & -39 & -12 & $-0.009(0.131)$ & $0.228(0.143)$ \\
\hline & MTG.R & 10 & 69 & -42 & 3 & $-0.194(0.175)$ & $0.074(0.188)$ \\
\hline control < SPD & MFG.R & 28 & 39 & 39 & 33 & $0.217(0.229)$ & $-0.083(0.122)$ \\
\hline \multicolumn{8}{|l|}{ PCUN.L } \\
\hline \multirow[t]{2}{*}{ control > SPD } & PHG.R & 6 & 36 & -33 & -15 & $0.008(0.138)$ & $0.258(0.178)$ \\
\hline & STG.R & 9 & 30 & -57 & 27 & $-2.843(0.181)$ & $0.252(0.174)$ \\
\hline
\end{tabular}

SPD schizotypal personality disorder, PCUN.R right precuneus, PHG.L left parahippocampus, PHG.R right parahippocampus, MTG.R right middle temporal gyrus, MFG.R right middle frontal gyrus, PCUN.L left precuneus, $P H G . R$ right parahippocampus, STG.R right superior temporal gyrus

avoxel threshold $p<0.001$, cluster threshold $p<0.05$ using Alphasim correction 


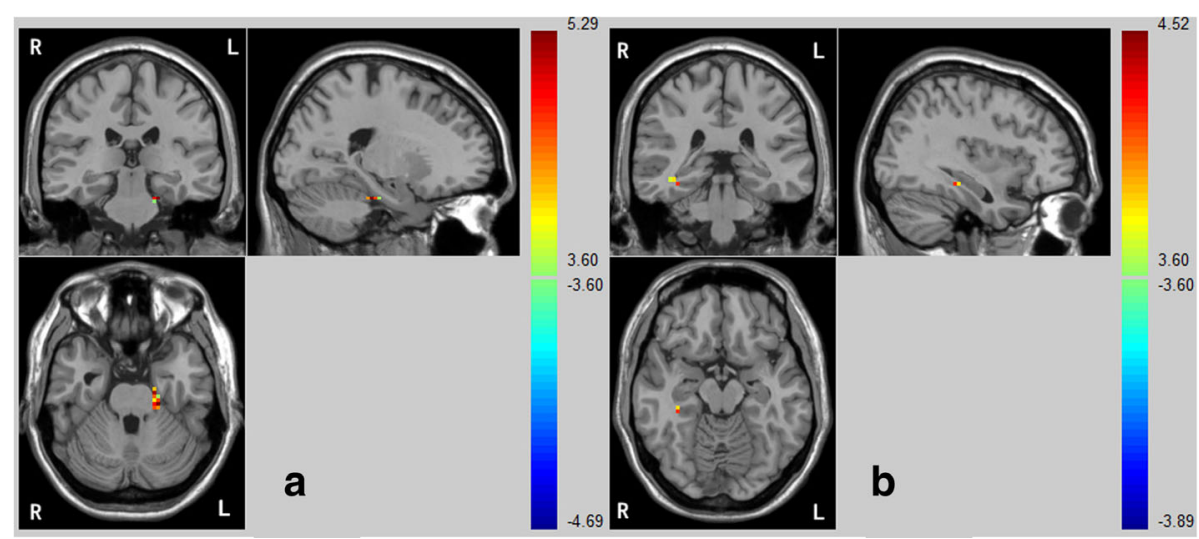

Fig. 1 Compared with the control group, the SPD group exhibited decreased FC between the bilateral precuneus and opposite lateral (a: left; b: right) parahippocampus

in unmedicated patients with schizophrenia, and they demonstrated that the peak area of FC deficits between the hippocampus and precuneus in schizophrenic patients lies within the central area of the precuneus that is thought to be relevant for cognition. Our results are in consistency with these findings and demonstrate more prominent schizotypal traits are linked with more remarkable hippocampal-parietal dysconnectivity. Suspiciousness is categorized as cognitive perceptual disturbance according to the higher-order models for SPQ [40, 48]. It is also involved in the social cognition and metacognition, for instance, the evaluation and estimation on others according to common sense.

Moreover, we also found that a reduced FC between left precuneus and right parahippocampus was inversely correlated with SPQ subscale score of suspiciousness in
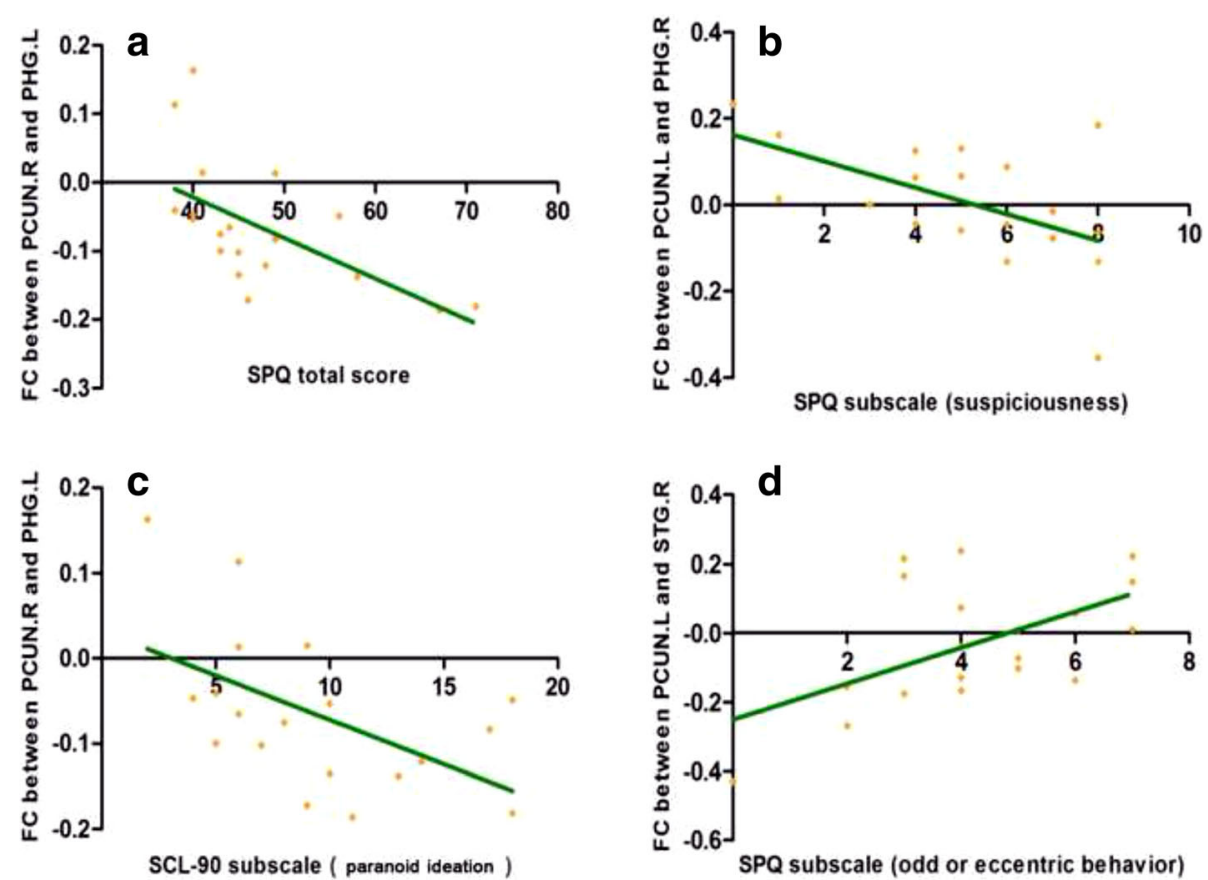

Fig. 2 In SPD group, higher SPQ total score was found to be negatively associated with FC between the right precuneus and left parahippocampus $(r=-0.603, p=0.006)(\mathbf{a})$; there was a negative trend between SPQ subscore (suspiciousness) and FC between the left precuneus and right parahippocampus $(r=-0.553, p=0.014)(\mathbf{b})$; a similar negative trend was found between SCL-90 subscore (paranoid ideation) and FC between the right precuneus and left parahippocampus $(r=-0.535, p=0.018)(\mathbf{c})$; a positive trend was found between SPQ subscore (odd or eccentric behavior) and FC between left precuneus and right superior temporal gyrus $(r=0.543, p=0.016)(\mathbf{d})$. Abbreviation: PCUN.R, right precuneus; PHG.L, left parahippocampus; PCUN.L, left precuneus; PHG.R, right parahippocampus; STG.R, right superior temporal gyrus; SPQ, Schizotypal Personality Questionnaire; SCL-90, Symptom Checklist 
SPD group. These results further supported the associations of FC between precuneus and parahippocampus with schizotypal traits. Previous studies supported that abnormal FC between precuneus and parahippocampus possibly plays a role in the continuum from individuals with schizotypal personality traits to developing schizophrenia. For instance, Allen et al. [49] recently reported that people at clinical high risk for psychosis showed parahippocampal hypoactivation during memory encoding. The authors also found that the parahippocampal response was correlated with the level of striatal dopamine function in the clinical high-risk individuals, which was absent in controls. Thus, they proposed that parahippocampal hypoactivation is related to an increased vulnerability to schizophrenia [49].

Several limitations should be considered while interpreting our results. First, the small sample size reduced detecting power, leading that the corrected clusters were relatively small. Second, our results only found a continuum of FC alteration in the individuals with schizotypal personality disorder and its direct validation in schizophrenia patients is still needed. Lastly, we did not detect FC of each subdivision in precuneus, which has been demonstrated to be involved in different functions $[16,17]$. Therefore, we would like to keep our findings in the present study as preliminary.

\section{Conclusion}

Taken together, our findings demonstrated that the functional connectivity between precuneus and parahippocampus, between precuneus and middle/superior temporal gyrus, might play a key role in the pathophysiology of schizophrenia spectrum disorder.

\section{Abbreviation}

CSF: Cerebral spinal fluid; FC: Functional connectivity; FOV: Field of view; FWHM: Full width at half-maximum; MNI: Montreal neurological institute; MRI: Magnetic resonance imaging; ROls: Region-of-interest; rs-fMRI: Restingstate functional MRI; SCID-II: Structured clinical interview for DSM-IV Axis II; SCL-90: Symptom checklist; SPD: Schizotypal personality disorder;

SPQ: Schizotypal personality questionnaire; TE: Echo time; TR: Repetition time

\section{Acknowledgments}

We would like to thank all the participants in this study.

\section{Funding}

Design and data collection of this work were supported by grants from National Natural Science Foundation of China $(81201043,81171267$, $81171280,81261120410,81372122,81361120403,81471359)$, Shanghai Science and Technology Committee (15411967200, 14411961400, 134119b0300, 15ZR1435500, 14411963400, 16XD1402400), Shanghai Sailing Program (16YF1416000), National Key Clinical Disciplines at Shanghai Mental Health Center (OMA-MH, 2011-873), Shanghai Key Laboratory of Psychotic Disorders (13dz2260500). Data analysis and interpretation was supported by Shanghai Jiao Tong University Foundation (14JCRY04, YG2014MS40), SHSMU-ION Research Center for Brain Disorders (2015NKX001, 15ZH2015), Shanghai Health System Advanced Appropriate Technology (2013SY003) and The Medicine Engineering Intersection Program of Shanghai Jiao Tong University (YG2015ZD12), Research Grant from Shanghai Hospital Development Center (SHDC12014111). Writing the manuscript was supported by Shanghai Municipal Commission of Health and Family Planning (20144Y0053), Shanghai
Mental Health Center (2014-FX-01, 2013-YJGJ-05, 2013-QH-02, 2013-YJTSZK-05). Yikang Zhu was supported by 2014 Shanghai Jiao Tong University SMC-Morning Star Award for Young Investigator, the Shanghai Jiao Tong University K. C. Wong Medical Fellowship Fund and visiting program of Shanghai Municipal Education Commission. The funder had no role in the design, collection, management, analysis, and approval of manuscript or decision to submit the manuscript for publication.

\section{Availability of supporting data}

All data generated or analysed during this study are included in the figures in this published article.

\section{Authors' contributions}

WJJ, LCB, TYX, LXG, HYG and LZ contributed to the overall design. LH, TYY, $Z T H, H Y G$ and $L Z$ involved in sample collection and clinical diagnosis validation. ZYK, ZTH and LXG undertook the statistical analysis and interpretation of data. ZYK, ZTH, LH, LCB and WJJ wrote the draft of the manuscript. WJJ, LZ, HYG and $L X G$ reviewed the manuscript and contributed to the interpretation of data in the revised manuscript. All authors have contributed to approve the final manuscript.

\section{Competing interests}

The authors declare that they have no competing interests.

\section{Consent to publish}

Not applicable.

\section{Ethics approval and consent to participate}

The study was approved by the local institutional ethics board (Institutional Review Board of Shanghai Mental Health Center, Shanghai Jiao Tong University School of Medicine) and carried out in accordance with the Declaration of Helsinki. Written informed consent was received from all participants prior to inclusion.

\section{Author details}

'Shanghai Key Laboratory of Psychotic Disorders, Shanghai Mental Health Center, Shanghai Jiao Tong University School of Medicine, South Wan Ping Road 600, Shanghai 200030, People's Republic of China. ${ }^{2}$ Klinik und Poliklinik für Psychiatrie und Psychotherapie, Klinikum rechts der Isar, TU München, Munich, Germany. ${ }^{3}$ Department of Medical Psychology, Faculty of Psychology and Mental Health, Second Military Medical University, Shanghai, People's Republic of China. ${ }^{4}$ Department of Psychiatry, Yale University School of Medicine, New Haven, CT 06516, USA. ${ }^{5}$ Department of Psychiatry, Shanghai Tongji Hospital, Tongji University School of Medicine, 389 Xin Cun Road, Shanghai 200065, People's Republic of China. ${ }^{6}$ Bio-X Institutes, Key Laboratory for the Genetics of Developmental and Neuropsychiatric Disorders, Ministry of Education, Shanghai Jiao Tong University, Shanghai, People's Republic of China.

Received: 31 May 2016 Accepted: 29 November 2016 Published online: 02 February 2017

\section{References}

1. Association AP. Diagnostic and statistical manual of mental disorders (DSM-5). Arlington: American Psychiatric Publishing; 2013.

2. Siever $\amalg$, Koenigsberg HW, Harvey P, Mitropoulou V, Laruelle M, Abi-Dargham A, Goodman M, Buchsbaum M. Cognitive and brain function in schizotypal personality disorder. Schizophr Res. 2002:54(1-2):157-67.

3. Hazlett EA, Lamade RV, Graff FS, McClure MM, Kolaitis JC, Goldstein KE, Siever LJ, Godbold JH, Moshier E. Visual-spatial working memory performance and temporal gray matter volume predict schizotypal personality disorder group membership. Schizophr Res. 2014;152(2-3):350-7.

4. McClure MM, Barch DM, Flory JD, Harvey PD, Siever LJ. Context processing in schizotypal personality disorder: evidence of specificity of impairment to the schizophrenia spectrum. J Abnorm Psychol. 2008;117(2):342-54.

5. McClure MM, Harvey PD, Bowie CR, lacoviello B, Siever LJ. Functional outcomes, functional capacity, and cognitive impairment in schizotypal personality disorder. Schizophr Res. 2013;144(1-3):146-50.

6. Mitropoulou V, Harvey PD, Maldari LA, Moriarty PJ, New AS, Silverman JM, Siever LJ. Neuropsychological performance in schizotypal personality 
disorder: evidence regarding diagnostic specificity. Biol Psychiatry. 2002;52(12):1175-82.

7. Siever LJ, Davis KL. The pathophysiology of schizophrenia disorders: perspectives from the spectrum. Am J Psychiatry. 2004;161(3):398-413.

8. Alonso-Solis A, Corripio I, de Castro-Manglano P, Duran-Sindreu S, GarciaGarcia M, Proal E, Nunez-Marin F, Soutullo C, Alvarez E, Gomez-Anson B, et al. Altered default network resting state functional connectivity in patients with a first episode of psychosis. Schizophr Res. 2012;139(1-3):13-8.

9. Borgwardt SJ, McGuire PK, Aston J, Berger G, Dazzan P, Gschwandtner U, Pfluger M, D'Souza M, Radue EW, Riecher-Rossler A. Structural brain abnormalities in individuals with an at-risk mental state who later develop psychosis. Br J Psychiatry. 2007;191:S69-75.

10. Gong XH, Lu WL, Kendrick KM, Pu WD, Wang C, Jin L, Lu GM, Liu ZN, Liu $\mathrm{HH}$, Feng JF. A brain-wide association study of DISC1 genetic variants reveals a relationship with the structure and functional connectivity of the precuneus in schizophrenia. Hum Brain Mapp. 2014;35(11):5414-30.

11. Guo Q, Tang YY, Li H, Zhang TH, Li JQ, Sheng JH, Liu DT, Li CB, Wang JJ. Both volumetry and functional connectivity of Heschl's gyrus are associated with auditory P300 in first episode schizophrenia. Schizophr Res. 2014;160(1-3):57-66.

12. Kindler J, Jann K, Homan P, Hauf M, Walther S, Strik W, Dierks T, Hubl D. Static and dynamic characteristics of cerebral blood flow during the resting state in schizophrenia. Schizophr Bull. 2015;41(1):163-70.

13. Mashal N, Vishne T, Laor N. The role of the precuneus in metaphor comprehension: evidence from an fMRI study in people with schizophrenia and healthy participants. Front Hum Neurosci. 2014:8.

14. Yang Z, Xu Y, Xu T, Hoy CW, Handwerker DA, Chen G, Northoff G, Zuo XN Bandettini PA. Brain network informed subject community detection in early-onset schizophrenia. Scientific Reports. 2014;4:5549.

15. Cavanna AE, Trimble MR. The precuneus: a review of its functional anatomy and behavioural correlates. Brain. 2006;129:564-83.

16. Margulies DS, Vincent JL, Kelly C, Lohmann G, Uddin LQ, Biswal BB, Villringer A, Castellanos FX, Milham MP, Petrides M. Precuneus shares intrinsic functional architecture in humans and monkeys. Proc Natl Acad Sci U S A. 2009;106(47): 20069-74

17. Zhang S, Li CSR. Functional connectivity mapping of the human precuneus by resting state fMRI. Neuroimage. 2012;59(4):3548-62.

18. Fransson P, Marrelec $\mathrm{G}$. The precuneus/posterior cingulate cortex plays a pivotal role in the default mode network: Evidence from a partial correlation network analysis. Neuroimage. 2008;42(3):1178-84.

19. Utevsky AV, Smith DV, Huettel SA. Precuneus is a functional core of the default-mode network. J Neurosci. 2014;34(3):932-40.

20. Yang Z, Chang C, X T, Jiang LL, Handwerker DA, Castellanos FX, Milham MP, Bandettini PA, Zuo XN. Connectivity trajectory across lifespan differentiates the precuneus from the default network. Neuroimage. 2014;89:45-56.

21. Frings L, Dressel K, Abel S, Saur D, Kummerer D, Mader I, Weiller C, Hull M. Reduced precuneus deactivation during object naming in patients with mild cognitive impairment, Alzheimer's disease, and frontotemporal lobar degeneration. Dement Geriatr Cogn Disord. 2010;30(4):334-43.

22. Kapogiannis D. Precuneus Glucose Reflects CSF A beta 42 in Early Alzheimer's Disease. Neurol. 2013;80(7):Supplement P06.043.

23. Karas G, Scheltens P, Rombouts S, van Schijndel R, Klein M, Jones B, van der Flier W Vrenken $H$, Barkhof F. Precuneus atrophy in early-onset Alzheimer's disease: a morphometric structural MRI study. Neuroradiology. 2007:49(12):967-76.

24. Kim J, Kim YH, Lee JH. Hippocampus-precuneus functional connectivity as an early sign of Alzheimer's disease: a preliminary study using structural and functional magnetic resonance imaging data. Brain Res. 2013;1495:18-29.

25. Maarouf $\mathrm{CL}$, Kokjohn TA, Walker DG, Whiteside CM, Kalback WM, Whetzel A, Sue LI, Serrano G, Jacobson SA, Sabbagh MN, et al. Biochemical Assessment of Precuneus and Posterior Cingulate Gyrus in the Context of Brain Aging and Alzheimer's Disease. PLoS One. 2014;9(8):e105784. doi:10.1371/journal. pone.0105784. eCollection 2014.

26. Nelson PT, Abner EL, Scheff SW, Schmitt FA, Kryscio RJ, Jicha GA, Smith CD, Patel E, Markesbery WR. Alzheimer's-type neuropathology in the precuneus is not increased relative to other areas of neocortex across a range of cognitive impairment. Neurosci Lett. 2009;450(3):336-9.

27. Perez SE, He B, Nadeem M, Wuu J, Scheff SW, Abrahamson EE, Ikonomovic MD, Mufson EJ. Resilience of precuneus Neurotrophic signaling pathways despite amyloid pathology in prodromal Alzheimer's disease. Biol Psychiatry. 2015;77(8):693-703.

28. Rami L, Sala-Llonch R, Sole-Padulles C, Fortea J, Olives J, Llado A, Pena-Gomez C, Balasa M, Bosch B, Antonell A, et al. Distinct functional activity of the precuneus and posterior cingulate cortex during encoding in the preclinical stage of Alzheimer's disease. J Alzheimers Dis. 2012;31(3):517-26.

29. Scheff SW, Price DA, Schmitt FA, Roberts KN, Ikonomovic MD, Mufson EJ. Synapse stability in the precuneus early in the progression of Alzheimer's disease. J Alzheimers Dis. 2013;35(3):599-609.

30. Stricker NH, Dodge HH, Dowling NM, Han SD, Erosheva EA, Jagust WJ, Neuroimaging AsD. CSF biomarker associations with change in hippocampal volume and precuneus thickness: implications for the Alzheimer's pathological cascade. Brain Imaging Behav. 2012;6(4):599-609.

31. Delaveau P, Jabourian M, Lemogne C, Choucha W, Girault N, Lehericy S, Laredo J, Fossati P. Dorsomedial and Precuneus Activation during Self Referential Processing Predict Long Term Remission to Agomelatine in Major Depression. Munich: Presented at the European Psychiatric Association Congress; March 1-4, 2014. e-poster EPA-1086.

32. Dumas R, Richieri R, Guedj E, Auquier P, Lancon C, Boyer L. Improvement of health-related quality of life in depression after transcranial magnetic stimulation in a naturalistic trial is associated with decreased perfusion in precuneus. Health Qual Life Outcomes. 2012;10:87.

33. Fossati P. The eye of the self: precuneus and depression. Munich: Presented at the European Psychiatric Association Congress; March 1-4, 2014. e-poster EPA-1043.

34. Kito S, Pascual-Marqui RD, Hasegawa T, Koga Y. High-frequency left prefrontal transcranial magnetic stimulation modulates resting EEG functional connectivity for gamma band between the left dorsolateral prefrontal cortex and precuneus in depression. Brain Stimul. 2014;7(1):145-6.

35. Peng D, Liddle EB, Iwabuchi SJ, Zhang C, Wu Z, Liu J, Jiang K, Xu L, Liddle $P F$, Palaniyappan $L$, et al. Dissociated large-scale functional connectivity networks of the precuneus in medication-naive first-episode depression. Psychiatry Res Neuroimaging. 2015;232(3):250-6.

36. Zhu X, Wang X, Xiao J, Liao J, Zhong M, Wang W, Yao S. Evidence of a dissociation pattern in resting-state default mode network connectivity in first-episode, treatment-naive major depression patients. Biol Psychiatry. 2012;71(7):611-7.

37. Kraguljac NV, White DM, Hadley J, Reid MA, Lahti AC. Hippocampal-parietal dysconnectivity and glutamate abnormalities in unmedicated patients with schizophrenia. Hippocampus. 2014;24(12):1524-32.

38. Nenadic I, Lorenz C, Langbein K, Dietzek M, Smesny S, Schonfeld N, Fananas L, Sauer H, Gaser C. Brain structural correlates of schizotypy and psychosis proneness in a non-clinical healthy volunteer sample. Schizophr Res. 2015;168(1-2):37-43.

39. Raine A. The SPQ: a scale for the assessment of schizotypal personality based on DSM-III-R criteria. Schizophr Bull. 1991;17(4):555-64.

40. Raine A, Reynolds C, Lencz T, Scerbo A, Triphon N, Kim D. Cognitiveperceptual, interpersonal, and disorganized features of schizotypal personality. Schizophr Bull. 1994;20(1):191-201.

41. Rytila-Manninen M, Frojd S, Haravuori H, Lindberg N, Marttunen M, Kettunen K, Therman S. Psychometric properties of the Symptom Checklist90 in adolescent psychiatric inpatients and age- and gender-matched community youth. Child Adolesc Psychiatr Ment Health. 2016;10:23.

42. Li H, Chen ZT, Tang YX, Li CB, Xiao ZP, Yin F, Wang JJ. Social cognition of patients with schizotypal personality disorder. Chin Ment Health J. 2010;24:270-4.

43. Anticevic A, Repovs G, Barch DM. Working memory encoding and maintenance deficits in schizophrenia: neural evidence for activation and deactivation abnormalities. Schizophr Bull. 2013;39(1):168-78.

44. Palaniyappan $L$, Simmonite M, White TP, Liddle EB, Liddle PF. Neural primacy of the salience processing system in schizophrenia. Neuron. 2013;79(4):814-28.

45. Whitfield-Gabrieli S, Thermenos HW, Milanovic S, Tsuang MT, Faraone SV, McCarley RW, Shenton ME, Green Al, Nieto-Castanon A, LaViolette P, et al. Hyperactivity and hyperconnectivity of the default network in schizophrenia and in first-degree relatives of persons with schizophrenia. Proc Natl Acad Sci U S A. 2009;106(4):1279-84.

46. Woodward TS, Leong K, Sanford N, Tipper CM, Lavigne KM. Altered balance of functional brain networks in Schizophrenia. Psychiatry Res. 2016;248:94-104.

47. Xin F, Lei X. Competition between frontoparietal control and default networks supports social working memory and empathy. Soc Cogn Affect Neurosci. 2015;10(8):1144-52.

48. Davidson CA, Hoffman L, Spaulding WD. Schizotypal personality questionnaire - brief revised (updated): an update of norms, factor structure, and item content in a large non-clinical young adult sample. Psychiatry Res. 2016;238:345-55.

49. Allen P, McGuire PK. Parahippocampal hypoactivation and vulnerability to schizophrenia. JAMA Psychiatry. 2014;71(11):1300-1. 